\title{
« Hegel et Husserl sur l'intersubjectivité » : la genèse longue d'un écrit de circonstance
}

\section{Roberta Picardi et Chiara Pavan}

\section{(2) OpenEdition}

1 Journals

Édition électronique

URL : https://journals.openedition.org/genesis/1798

DOI : 10.4000/genesis. 1798

ISSN : 2268-1590

Éditeur :

Presses universitaires de Paris Sorbonne (PUPS), Société internationale de génétique artistique littéraire et scientifique (SIGALES)

Édition imprimée

Date de publication : 9 mai 2017

Pagination : 203-212

ISBN : 979-1023-105636

ISSN : 1167-5101

\section{Référence électronique}

Roberta Picardi et Chiara Pavan, « « Hegel et Husserl sur l'intersubjectivité » : la genèse longue d'un écrit de circonstance », Genesis [En ligne], 44 | 2017, mis en ligne le 22 mai 2018, consulté le 17 mars 2023. URL : http://journals.openedition.org/genesis/1798; DOI : https://doi.org/10.4000/genesis. 1798 


\title{
"Hegel et Husserl sur l'intersubjectivité»: la genèse longue d'un écrit de circonstance
}

\author{
Chiara Pavan et Roberta Picardi
}

$\mathrm{H}$ egel et Husserl sur l'intersubjectivité » est un essai de Paul Ricœur, qui fut inclus en 1986 dans le recueil Du texte à l'action, avec quelques remaniements par rapport à ses versions précédentes ${ }^{1}$. Il s'agit d'un écrit de circonstance que, selon toute probabilité, Ricœur rédige pour une conférence à la New School for Social Research à l'automne 1976, comme on peut l'inférer sur la base de documents conservés par David Pellauer, auteur de la traduction anglaise du texte ${ }^{2}$. Nous pourrions donc formuler l'hypothèse d'une genèse brève de cet essai, qui l'apparenterait à la plupart des écrits de circonstance. Mais ceci est démenti par l'étude des manuscrits conservés aux Archives du Fonds Ricœur, qui dévoilent au contraire une gestation dont les premières traces remontent jusqu'à dix ans avant la première publication du texte ${ }^{3}$.

La reconstruction de ce parcours génétique présente un double intérêt. Tout d'abord, cette genèse longue donne un aperçu très significatif de la manière dont Ricœur travaille, car elle permet de cerner les différentes étapes - souvent estompées dans les textes publiés - de son corps à corps spéculatif avec Husserl et Hegel, qui sont parmi les principaux auteurs dont se nourrissent son écriture et sa pensée. En outre, le parcours génétique de ce texte dévoile un itinéraire dont l'intérêt théorique est remarquable : c'est en effet la confrontation serrée avec Hegel et Husserl dont témoignent les manuscrits - qui conduit Ricœur à abandonner la conception de l'intersubjectivité défendue dans les écrits des années cinquante, dans lesquels il se réclame principalement de Kant, selon une orientation à la fois anti-hégélienne et anti-husserlienne. Ce n'est donc qu'à travers l'étude des documents de genèse qu'il devient possible de suivre pas à pas le mouvement non linéaire qui amène Ricœur à quitter la voie kantienne, en même temps qu'il réévalue et développe la conception husserlienne de l'intersubjectivité, afin de répondre au «défi» de l'Esprit objectif hégélien.
Dans cet article, nous nous proposons de mettre à jour ce mouvement. Dans ce but, après une brève description du dossier de genèse que nous avons reconstitué, nous nous concentrerons sur deux segments de la genèse de cet essai. En premier lieu, nous analyserons les notes manuscrites contenant les traces du questionnement qui aboutit à la conception du premier «schéma», à l'origine de l'essai «Hegel et Husserl sur l'intersubjectivité»; en deuxième lieu, nous aborderons les notes sur Husserl, notamment celles sur la «Cinquième méditation », rédigées entre les années soixante et les années soixante-dix, qui sous-tendent la textualisation de la partie centrale de l'essai.

1.Paul Ricœur, Du texte à l'action. Essais d'herméneutique II, Paris, Éditions du Seuil, coll. « Esprit», 1986, p. 281-302.

2. David Pellauer a conservé un fichier qui contient une photocopie du manuscrit original français et sa propre traduction : sur la chemise du dossier on peut lire : «lecture New School, 12 November 1976». Le brouillon français du texte - qui n'est pas daté et qui est intitulé «Phénoménologie hégélienne et phénoménologie husserlienne »- est conservé dans les Archives du Fonds Ricœur (Archives Ricœur, boîte 33, dossier 142, f. 17274-17296). L'édition anglaise du texte - qui précède sa publication en français - paraît dans le volume Reason, Action and Experience. Essays in Honor of Raymond Klibansky, éd. H. Kohlenberger, Hamburg, Meiner, 1979, p. 13-29. Le texte paraît successivement en français dans un volume collectif rassemblant les travaux de deux différentes sessions d'étude du CDRHM : à savoir Phénoménologies hégélienne et husserlienne. Les classes sociales selon Marx, éd. G. Planty-Bonjour, Paris, CNRS, 1981, p. 5-17.

3. Nous remercions vivement Mme Catherine Goldenstein - fondatrice des Archives Ricœur - pour son œil expert et son aide irremplaçable dans la recherche, la datation et la transcription des manuscrits. Pour toute information sur les Archives Ricoeur (Bibliothèque de l'Institut protestant de théologie), voir : < www.fondsricoeur.fr/fr/pages/accueil.html >. 


\section{Aperçu général des documents de genèse}

Les documents de genèse conservés dans le Fonds Ricœur - bien qu'ils ne soient pas complets - permettent néanmoins de suivre le processus de genèse de ce texte, de sa première conception et sa rédaction jusqu'à la mise au point avant publication.

On retrouve un polycopié intitulé «Hegel et Husserl sur l'intersubjectivité », en français et en anglais (traduit par David Pellauer), de quarante-cinq pages recto au format A4 ${ }^{4}$. Il s'agit probablement de deux polycopiés que Ricœur utilise dans un premier temps pour ses interventions orales - comme on peut le déduire de la mention «Draft - Not For Publication », inscrite sur la version anglaise ${ }^{5}-$, puis pour la publication. Ces polycopiés témoignent, de toute façon, d'un travail de finalisation post-rédactionnelle, car la rédaction du texte est documentée plutôt par le brouillon manuscrit intitulé «Phénoménologie hégélienne et phénoménologie husserlienne ${ }^{6}{ }$. Il s'agit d'un document manuscrit de vingt-trois pages recto, sur des feuilles blanches de format $\mathrm{A} 4$, écrites à l'encre bleue uniforme tout au long du document. Une marge est laissée sur la gauche et utilisée pour des corrections et des ajouts, significatifs mais pas très nombreux. Le brouillon est en effet relativement peu raturé et offre une version déjà très proche de la version polycopiée et du texte publié. Les principales modifications résident dans de petits remaniements de la structure de l'article et dans des coupures, qui ne répondent pas uniquement à des exigences d'espace, mais plutôt stylistiques - comme la recherche d'une conclusion efficace - ou à la volonté de détacher le «texte» de la circonstance particulière de sa genèse 7 .

Dans ce brouillon relativement «propre», la seule exception est constituée par les deux derniers feuillets (f. 17295-17296), dont la fonction est plutôt prérédactionnelle, car ils ne contiennent que de simples notes schématiques - introduites par la locution «Éclairer la Ve Méditation par M. Weber» - qui ont servi de base pour la textualisation de la dernière partie du brouillon. Ces deux feuillets n'épuisent pas du tout la phase prérédactionnelle de l'essai «Hegel et Husserl sur l'intersubjectivité », dont les traces se retrouvent plutôt dans une pluralité de documents génétiques de périodes et natures différentes. La toute première conception du «schéma » à l'origine de l'essai est en effet attestée par un groupe des feuillets manuscrits - à l'encre bleue et presque uniforme - conservés dans le classeur «Plans pour une nouvelle philosophie de la volonté», remontant à la fin des années soixante. Il s'agit précisément des feuillets 31577-31587, contenant une ébauche de textualisation de la deuxième partie - intitulée «Du discours de la décision au discours de l'action »- de la «nouvelle philosophie» de la volonté projetée par Ricœur à la fin des années soixante, mais jamais réalisée. L'élargissement de ce premier «schéma» - qui concerne exclusivement l'articulation entre la phénoménologie husserlienne de l'intersubjectivité et la dialectique hégélienne de l'Esprit - est ensuite documenté par les notes manuscrites pour la préparation d'un cours donné à Chicago en 1973, conservées dans le classeur 30, dont l'intitulé est «The Implications of Phenomenology for a Political Philosophy. Max Weber» (f. 32279-32316). On y trouve non seulement la première conception de l'idée d'un couplement entre la phénoménologie husserlienne de l'intersubjectivité de Husserl et la sociologie compréhensive de Weber (fig. 1), mais également le travail de documentation sur Wirtschaft et Gesellschaft (f. 32283-32287), sur lequel s'appuie la textualisation des pages conclusives du brouillon et du polycopié.

Les analyses qui préparent la textualisation de la première partie de l'essai - consacrée au chapitre «Geist» de la Phénoménologie de l'Esprit - peuvent être suivies à travers deux types de supports documentaires : d'une part, les notes manuscrites d'un cours donné à Chicago en 1978, conservées dans le dossier 90.18 intitulé «Phén. de l'Esprit.

4. « Hegel et Husserl sur l'intersubjectivité», Archives Ricœur, Classeur 19, f. 31820-31822 (seule la première page des deux documents est numérotée).

5.Ibid., f. 31822, p. 1.

6. «Phénoménologie hégélienne et phénoménologie husserlienne », Archives Ricœur, boîte 33, dossier 142.2, f. 17274-17296.

7. Dans le polycopié comme dans le texte, Ricœur coupe par exemple les sept dernières lignes de la première partie, en concluant cette dernière par la phrase - à la fois suggestive et dense - «Cela même qui, avec Kant, s'était érigé en tribunal naît dans la douleur de l'arrachement ». Ce choix implique néanmoins la suppression d'une phrase qui exprime de façon claire un point central pour comprendre le rapprochement que Ricœur opère entre Hegel et Husserl : «[...] C'est pourquoi on est tenté de dire que ce qui s'appellera intenn/té chez H. s'appelle aliénation, dessaisissement chez Hegel». 
«HEGEL ET HUSSERL SUR L'INTERSUBJECTIVITÉ» : LA GENÈSE LONGUE D’UN ÉCRIT DE CIRCONSTANCE

$\begin{array}{lllll}V & A & R & I & A\end{array}$

32282

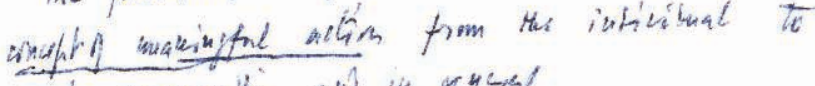

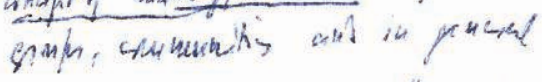

Un Max bieler as the exponaut if las atruim.

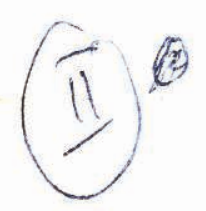

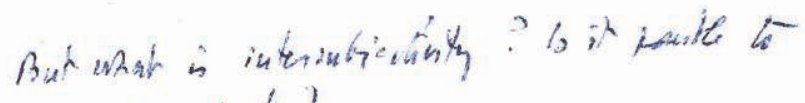
think if uhimileg?

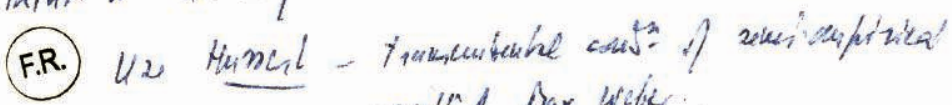
vouphl Max welic:

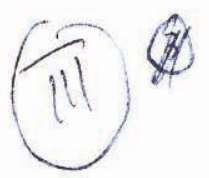

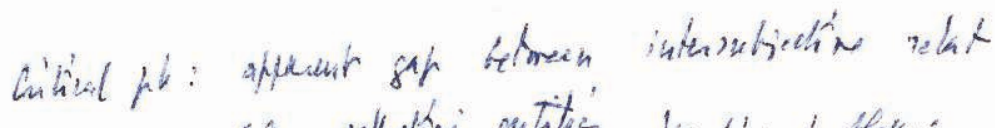

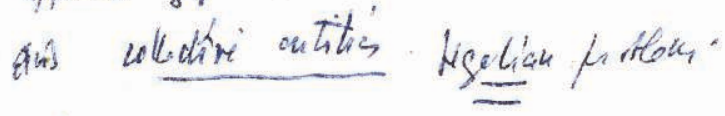

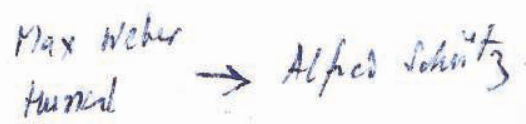

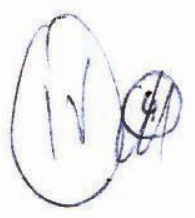

The earigus of authinity. Sruinath

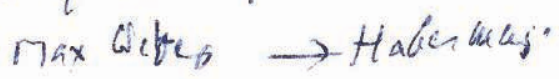

Fig. 1 : Paul Ricœur, feuillet extrait du dossier «The Implications of Phenomenology for a Political Philosophy. Max Weber» (Fonds Paul Ricœur, classeur 30, f. 32282) @ Comité éditorial du Fonds Ricœur

205 
Chap. VI» (f. 13015-13024) ${ }^{8}$; d'autre part, les annotations de Ricœur sur sa propre copie de la Phénoménologie de l'Esprit ${ }^{9}$, sur le commentaire de Hyppolite 10 et sur l'ouvrage de Labarrière ${ }^{11}$, qui peuvent être consultées à la bibliothèque du Fonds Ricœur. En revanche, en ce qui concerne la rédaction de la partie centrale de l'essai, qui porte sur la phénoménologie husserlienne, il est intéressant de se référer à un dossier de notes manuscrites intitulé : «V Méditation» (classeur 33, dossier 98, f. 13660-13712). Quoique ce dossier ne puisse pas être inclus à proprement parler dans la phase prérédactionnelle, il est important en ce qu'il montre la stratification dans la lecture de la théorie husserlienne de l'intersubjectivité, qui aboutit à la prise de position de l'essai «Hegel et Husserl sur l'intersubjectivité». Notamment, on y trouve une première partie (f. 13660-13707) dans laquelle les notes du début des années cinquante sont intercalées avec de nouvelles notes remontant à la période de la Sorbonne (entre la fin des années cinquante et le début des années soixante), qui se distinguent surtout par la couleur jaunie des feuilles lignées; une deuxième partie (f. 13708-13712), qui correspond à la phase rédactionnelle de l'essai «La cinquième Méditation cartésienne 12 », est constituée de feuilles blanches, écrites à l'encre bleue et très raturées, surtout pour des questions stylistiques. De l'avant-texte de notre dossier de genèse font partie, au contraire, le manuscrit, le polycopié et également le texte de 1975 intitulés «Analogie et intersubjectivité chez Husserl 13 ».

\section{La genèse d'une question}

Dans la première page du brouillon «Phénoménologie hégélienne et husserlienne», on peut remarquer la rature suivante : «Je me suis posé une question» (f. 17275). Cette phrase raturée - à la première personne - dévoile l'urgence personnelle de la question au centre du texte : à savoir, la question de vérifier si la phénoménologie husserlienne réussit à faire l'économie du concept hégélien d'Esprit objectif, en rendant raison de la réalité du monde spirituel, sans recourir à une entité collective supérieure à la conscience. Comme Ricœur le précise, il s'agit d'une question qui porte sur Husserl, mais qui «ne serait pas posée s'il n'y avait pas eu Hegel» (f. 17275 , p. 281). Si on lit cette précision à la lumière de la rature mentionnée, on peut soupçonner que même dans ces passages Ricœur ne se réfère pas seulement à une filiation historique - à savoir le fait que Husserl hérite de Hegel, à travers la médiation de Dilthey, le problème de la constitution du monde de l'esprit 14 - mais également au mouvement de sa propre pensée : à savoir, le questionnement autocritique sur les limites de la phénoménologie husserlienne, que la confrontation serrée avec la «dialectique hégélienne» de l'Esprit - entreprise dans les années soixante - contribue à alimenter de façon déterminante.

Cette hypothèse est confirmée par l'étude du dossier déjà mentionné, «Plans pour une nouvelle philosophie

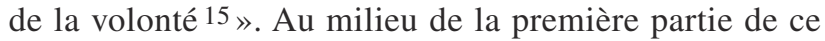

8. Il s'agit de notes écrites sur feuillets recto, avec différentes encres (verte, noire, bleu foncé et rouge), signe du fait que Ricœur les a utilisées pour plusieurs cours - à Chicago entre les années soixante-dix et quatrevingt - en les corrigeant et en les retravaillant : certaines formulations sont reprises presque littéralement dans le brouillon, bien qu'elles soient coupées dans le polycopié et dans le texte.

9. Copie annotée de G.F.W. Hegel, La Phénoménologie de l'Esprit, t. II, trad. J. Hyppolite, Paris, Aubier, 1941, cote G 193.34 HEG ph (2).

10. Copie annotée de J. Hyppolite, Genèse et structure de la Phénoménologie de l'Esprit de Hegel, Paris, Montaigne, 1946 (bibliothèque du Fonds Ricœur, cote F 193.33 HYP ge).

11. Copie annotée du livre de Pierre-Jean Labarrière, Structure et mouvement dialectique dans la Phénoménologie de 1'Esprit, Paris, Aubier, 1968 (bibliothèque du Fonds Ricœur, cote G 193.34 LAB).

12. La première édition de ce texte est en anglais : «Husserl's Fifth Cartesian Meditation », dans Husserl. An Analysis of his Phenomenology, Evanston, Northwestern University Press, 1967, p. 115-142.

13. Le manuscrit et le polycopié se trouvent aux Archives Ricœur, boîte 34, respectivement f. 17745-17753 et f. 17754-17774, datés de juin 1974. Le texte - intitulé «Analogie et intersubjectivité chez Husserl d'après les inédits de la période 1905-1920 (Éditions Iso Kern, Husserliana, t. XIII, Nijhoff, 1973)», a été publié dans Enige facetten van opvoeding en onderwijs, éd. A. Monshouwer, Malmberg, Den Bosch, 1975, p. 163-170. Malheureusement, nous n'avons pas trouvé, dans le Fonds Ricœur, la copie du tome XIII des Husserliana - édité par Iso Kern en 1973 et dédié à la question de l'intersubjectivité - sur laquelle Ricœur doit avoir travaillé pour la préparation de cet essai.

14. Cette filiation historique est néanmoins bien connue par Ricœur qui, dès les années cinquante, se concentre sur la troisième partie des Ideen II, dans laquelle Husserl traite notamment de la question de la constitution de la réalité spirituelle, dans le but programmatique d'offrir une fondation «radicale» à la distinction diltheyenne entre sciences de l'esprit et sciences de la nature (voir P. Ricœur, «Analyses et problèmes dans Ideen II de Husserl » (1952), À l'école de la phénoménologie, Paris, Vrin, 1986, p. 120-130).

15. Archives Ricœur, classeur 12 : «Plans pour une nouvelle philosophie de la volonté», f. 31534-31600. 
plan - intitulé «Les trois discours de la philosophie de la volonté»- se trouve en effet le propos de greffer une «dialectique de l'Esprit» (dont la filiation hégélienne est explicite) sur la phénoménologie de la décision d'inspiration husserlienne, qui lui semble inadéquate à rendre compte de la réalisation de la liberté dans le rapport à autrui, dans les normes et dans les institutions. De plus, dans cette ébauche, Ricœur indique que le facteur ayant déclenché ses doutes sur les «droits » et sur l' «extension» de la phénoménologie réside dans son étude de la troisième partie de l'Encyclopédie des sciences philosophiques, où la phénoménologie est réduite à un simple «moment» de la dialectique de l'esprit subjectif. De façon significative et surprenante, ce «recul» de la «phénoménologie» dans la philosophie hégélienne est ici valorisé comme un «avertiss. ${ }^{t}$ » que Hegel adresse «à t.te $\varphi$ én. ie qui voudrait $s$ 'égaler à $\varphi$. ie ḿ [même] » - de Kant à Husserl - et à toute prétention de réduire l'Esprit à la conscience et à l'intentionnalité qui la caractérise 16 . Dans les pages qui suivent, Ricœur va très loin dans cette direction, développant une lecture du «dépassement» du point de vue phénoménologique dans l'esprit subjectif et l'esprit objectif qui présente une évidente intention d'appropriation. En particulier, au début du feuillet 31587 (fig. 2), il écrit :

Comprendre la réalité présente ds. son essence raisonnable, voilà la $\varphi$. ie de la volonté. Et cette intelligence de la réalité n'est + phén.que, parce que cette réalité n'est + l'autre d'une cs, mais l'œuvre de l'E. Le rapport de l'E. à ses œuvres ne s'inscrit + ds. la dimension de l'intentionnalité.

Néanmoins, de façon très significative, c'est exactement à ce point de la page que, face à la prétention hégélienne d'intelligibilité totale suscitée par l'affirmation de l'identité entre l'Esprit et ses œuvres (et donc entre rationalité et réalité), Ricœur formule en marge le propos de reconsidérer la doctrine husserlienne de l'intersubjectivité, à la lumière de la thèse hégélienne sur l'excédence de l'Esprit par rapport à la dimension de la conscience et de l'intentionnalité : «confronter avec Husserl Ideen II, III et écrits sur la société ».

Cette note marginale exprime de façon tangible les hésitations de Ricœur dans sa confrontation avec Hegel. C'est dans ces hésitations que se fait jour la première idée dont l'essai «Hegel et Husserl sur l'intersubjectivité » est issu :

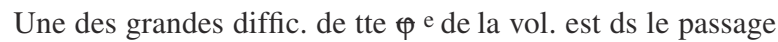
du discours sur la décision au discours sur l'action sensée. Car ce ne sont pas deux discours qui se succèdent, mais qui se croisent et s'interpénètrent. C'est la clé du rapport entre la

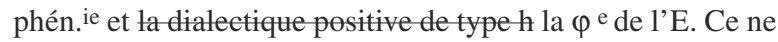
sont pas deux discours à mettre bout à bout $[. .$.$] .$

Il faudra traiter les 2 discours selon ce schéma : le $I^{\circ}$ s'achève plus loin que le $2^{\text {nd }}$ ne commence.

C'est le développement de ce «schéma» qui aboutit au texte «Hegel et Husserl sur l'intersubjectivité », où Ricœur explore de façon programmatique le «rapport croisé » entre la doctrine husserlienne de l'intersubjectivité et la dialectique hégélienne de l'Esprit. Dès lors, c'est l'exigence de respecter ce «schéma» d'articulation entre phénoménologie de la décision et dialectique de l'action, qui conduit Ricœur à choisir comme terrain de «rencontre» entre Husserl et Hegel non pas l'Encyclopédie mais plutôt la Phénoménologie de l'Esprit, dans laquelle la «ph. de l'E.» hégélienne, déployée dans le chapitre VI, demeure «dans l'e.[élément] de la conscience». Néanmoins, la manière dont Ricœur insiste, dans le brouillon et dans le texte, sur la différence entre la Phénoménologie et le système - dans lequel l'esprit objectif serait «hors phénoménologie»- ne doit pas nous conduire à oublier le point de départ du parcours génétique que l'on vient de reconstituer, à savoir, l' «avertissement» sur les limites de la phénoménologie et de l'intentionnalité, qui provient justement de ce «recul» de la phénoménologie dans l'Encyclopédie, dénoncé comme le vice du système dans le texte imprimé. En dépit de cette dénonciation, l'Encyclopédie des sciences philosophiques joue dans l'itinéraire intellectuel de Ricœur un rôle central - bien que souvent méconnu par les interprètes 17 -, ce dont les manuscrits offrent un témoignage clair.

16. Ricœur écrit ces passages en s'inspirant de façon explicite de la Remarque du paragraphe 415 de l'Encyclopédie, où Hegel attaque notamment la philosophie kantienne, en tant que «philosophie qui a conçu l'Esprit seulement comme conscience».

17. Voir par exemple l'article de O. Tinland, «La Phénoménologie de l'Esprit de Paul Ricœur», à paraître dans Ricœur et la philosophie allemande de Kant à Dilthey, dir. G. Marmasse et R. Picardi, Paris, CNRS Éditions, 2017. 
compinits ave thenel

Inem II, II it cuith us lo tricité

\section{2}

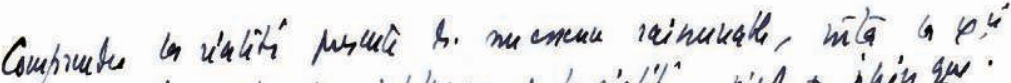

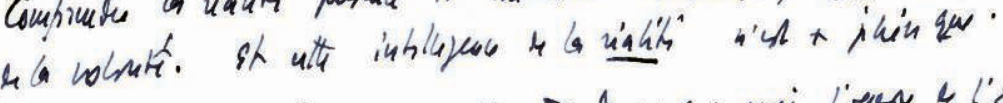

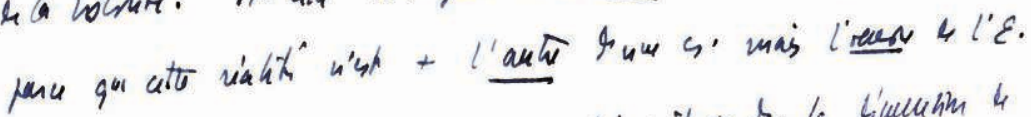

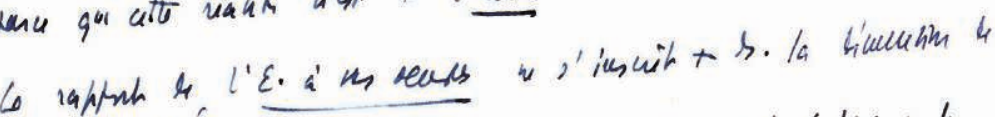
I' intentimality.

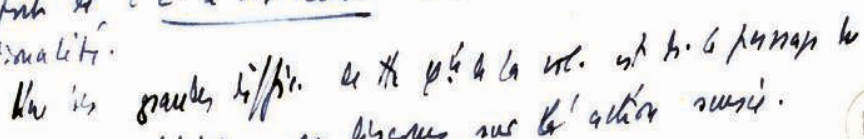

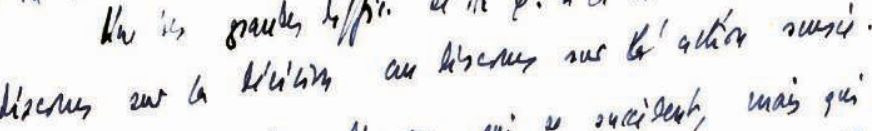

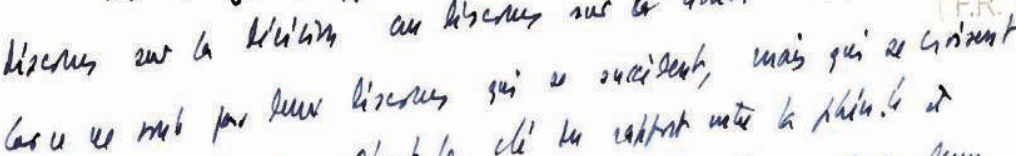

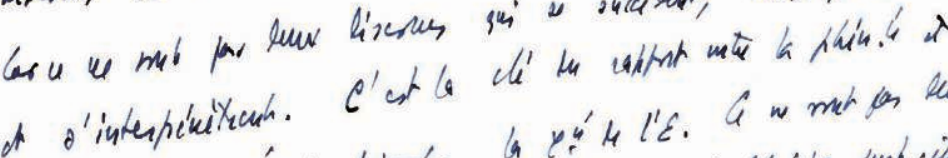

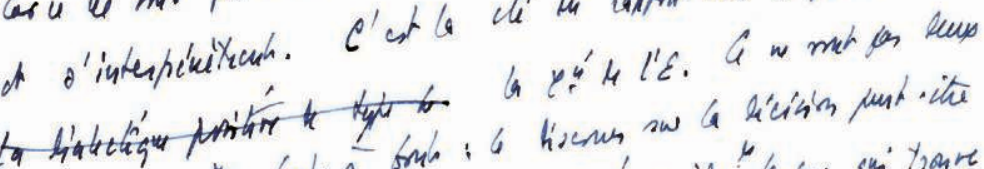

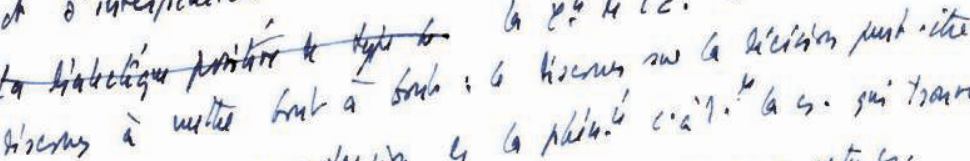

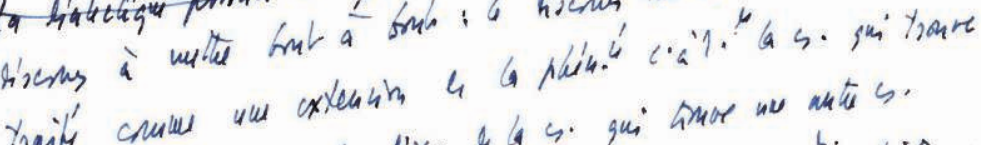

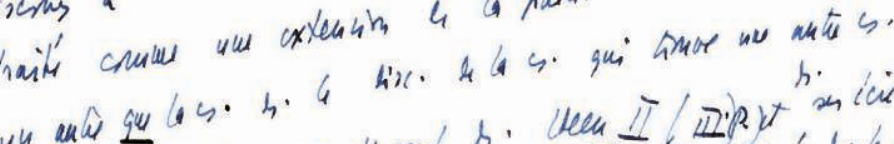

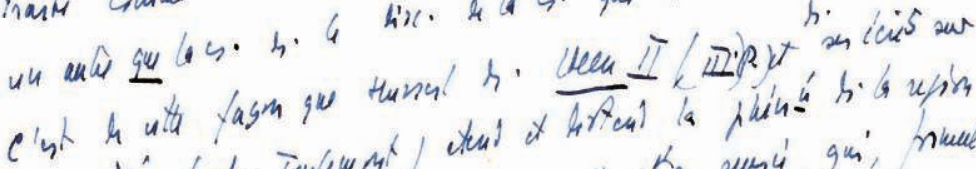

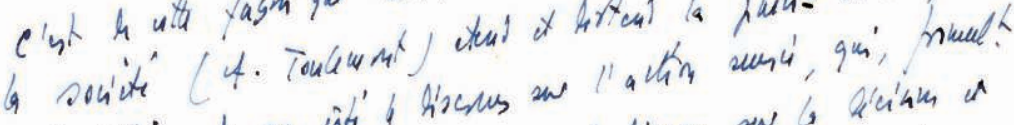

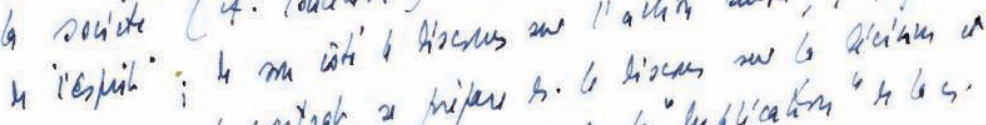

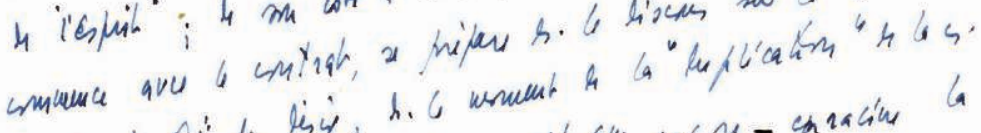

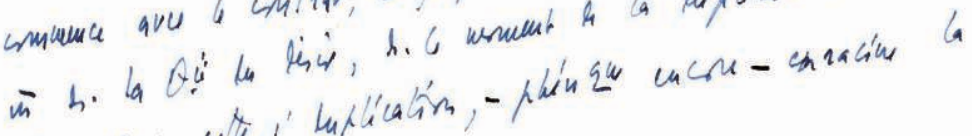

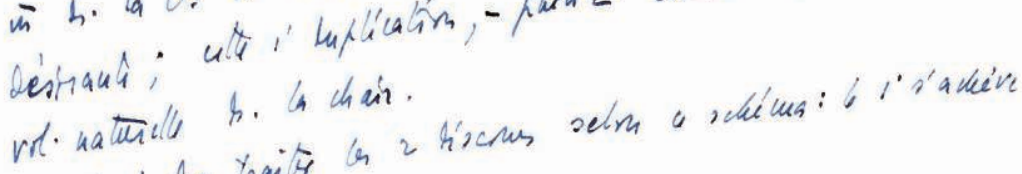

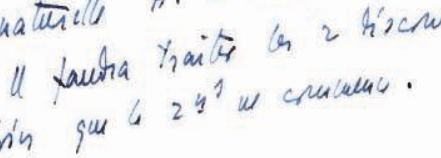
plos inin que 


\section{La Cinquième méditation : couches de lecture}

Comment Husserl peut-il prendre le relais du questionnement hégélien? Comment la théorie husserlienne de l'intersubjectivité peut-elle inscrire l'esprit objectif dans l'intentionnalité ? Ce sont ces questions qui émergent des notes examinées ci-dessus et qui motivent la rédaction de la partie centrale de l'essai. Or, cette nouvelle direction de recherche implique pour nous, lecteurs, une autre tâche, à savoir celle de comprendre comment Ricœur, après avoir accusé Husserl d'idéalisme subjectif et de solipsisme dans les années cinquante, en vient à adopter ici sa perspective transcendantale. Les manuscrits que nous avons étudiés nous fournissent des indications précieuses à cet égard.

Il est évident que si Ricœur introduit dans le brouillon «Phénoménologie hégélienne et phénoménologie husserlienne» (f. 17282) le paragraphe sur Husserl en précisant la signification du terme «constitution», c'est parce que ce concept a toujours été pour lui le point crucial et problématique de sa lecture de Husserl. Nous pouvons le voir dans plusieurs essais du début des années cinquante 18 : à cette époque, Ricœur estimait que, chez Husserl, la tension féconde entre la description de l'apparaître et la constitution du sens par l'ego, finissait par se déséquilibrer en faveur du deuxième élément - ce qui avait pour conséquence, dans la théorie de l'intersubjectivité, la réduction de l'altérité d'autrui à sa constitution par un ego transcendantal solipsiste. Or, les notes manuscrites des années soixante, portant sur la Cinquième méditation de Husserl, qui concerne justement la constitution de l'autre ${ }^{19}$, s'avèrent éclairantes pour comprendre le début de la transition vers l'essai de 1979 : Ricœur y approfondit les dynamiques de la saisie analogisante et commence à s'interroger sur la signification de la constitution du sens comme explicitation (Auslegung), mais il ne change pas d'avis sur la difficulté posée par la primauté de la constitution. Plutôt que la doctrine de l'analogie, c'est alors la notion plus générale de constitution qui demeure problématique aux yeux de Ricœur et qui l'empêche de reprendre, telle quelle, la théorie husserlienne de l'intersubjectivité. Le passage suivant en est un exemple (fig. 3) :

Subordinn ${ }^{\mathrm{n}}$ de la phénie descriptive à phén.ie fondative. Quel est finalt le sens de cette $\mathrm{V}^{\prime}$ Méd. par rapport à la tâche de la phén ${ }^{\text {ie }}$ Point le + extrême d'une lutte qui se poursuit depuis die Idee der Phänie (1907); je dirais : lutte entre le goût du concret au sens du total, et le goût l'exigence du radical au sens du principiel.

Voilà pourquoi, dans les années soixante-dix, lorsqu'il entend réhabiliter la doctrine husserlienne de l'intersubjectivité, Ricœur doit commencer par préciser le sens du terme «constitution», afin de fixer l'oscillation de ce terme entre la description d'un sens préexistant et la création du sens. Dans le brouillon «Phénoménologie hégélienne et phénoménologie husserlienne» (f. 17282), la question est tranchée. Ricour ne mentionne plus cette tension entre les deux exigences qu'en reconnaissant la présence de certains éléments allant dans la direction de l'idéalisme subjectif 20 ; mais, pour sa part, il interprète désormais la constitution comme Auslegung, c'est-à-dire comme un travail d'explicitation du sens, non point de création. Or, si Ricœur avoue lui-même que c'est grâce à l'herméneutique qu'il a pu voir dans la constitution une simple explicitation 21 , cette raison ne saurait être suffisante pour rendre compte du changement. S'il en était ainsi, cette position aurait dû déjà transparaître dans les notes des années soixante, postérieures à son tournant herméneutique. C'est en revanche la publication des inédits de Husserl sur l'intersubjectivité - édités par Iso Kern en $1973^{22}$ - qui s'avère déterminante pour l'interprétation ricœurienne de la constitution et de l'analogie : plutôt que d'être le point culminant de la tension entre la description et la constitution, laissant encore indéterminée la primauté de l'une ou de l'autre, l'analogie devient - après l'étude de ces textes - une co-position (Mitsetzung) du moi et de l'autre, co-position qui n' accorde aucune priorité au moi sur l'autre, et qui interrompt ainsi le parallèle problématique entre la constitution de la chose et celle de la personne que Husserl semblait instituer dans les Méditations. Nous

18. Tels que «Kant et Husserl» et «Sympathie et respect» (dans P. Ricœur, À l'école de la phénoménologie, Paris, Vrin, 2004, p. 273-313 et p. 333-359).

19. Notes prises en vue de la rédaction de l'essai de 1967, « Husserl's Fifth Cartesian Meditation » (art. cité).

20. Voir «Phénoménologie hégélienne et phénoménologie husserlienne», f. 17282 et P. Ricœur, «Hegel et Husserl sur l'intersubjectivité », art. cité, p. 289.

21. Ibid.

22. Voir notamment E. Husserl, Hua XIII, La Haye, M. Nijhoff, 1973. 


\section{$V_{24}$}

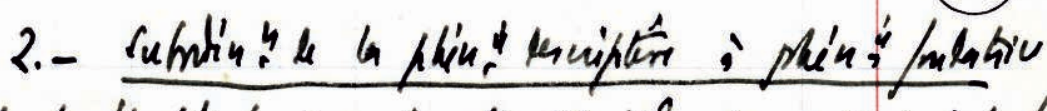

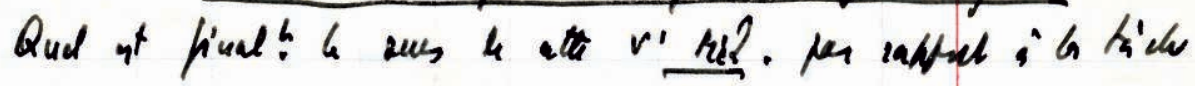

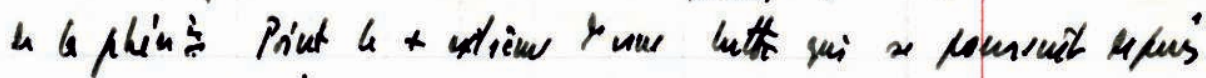

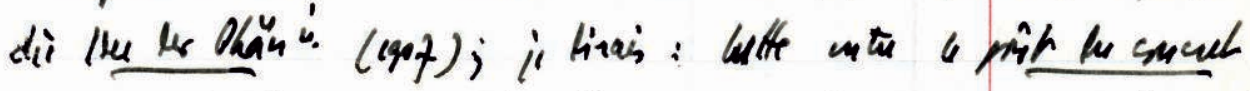

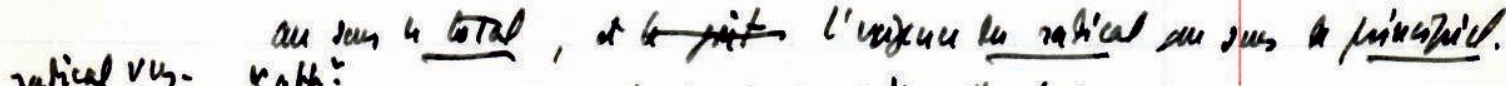

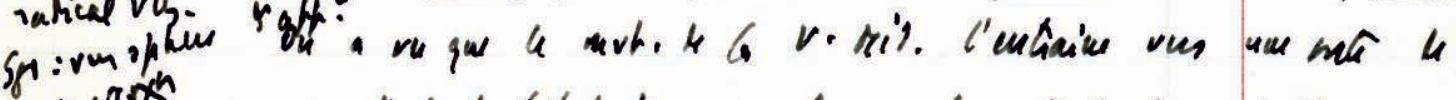

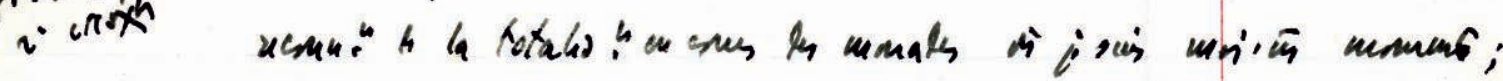

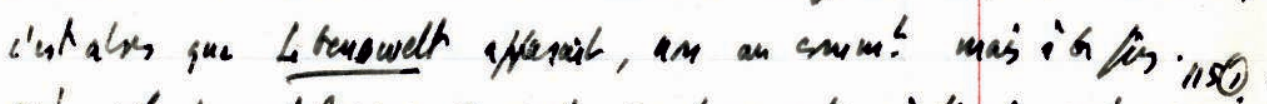

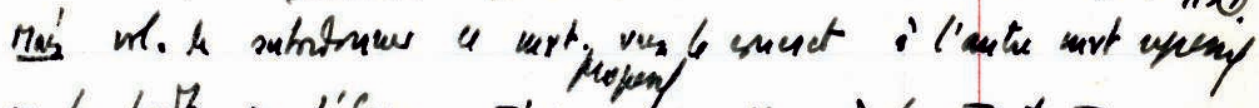

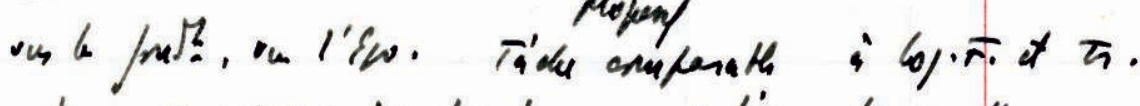

* $\rightarrow$ acterer 6/. frumle $\rightarrow$ y intiner bj.mats.

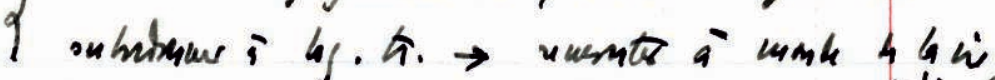

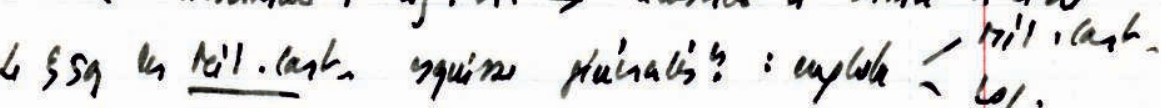

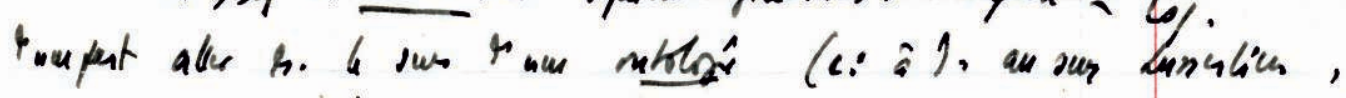

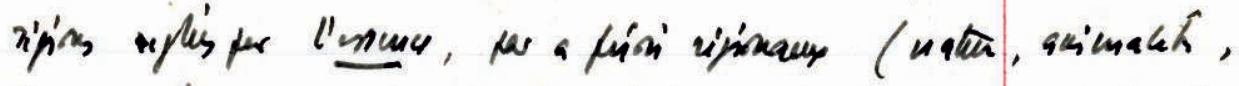

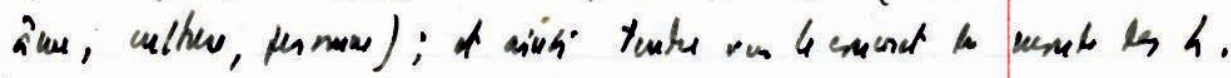

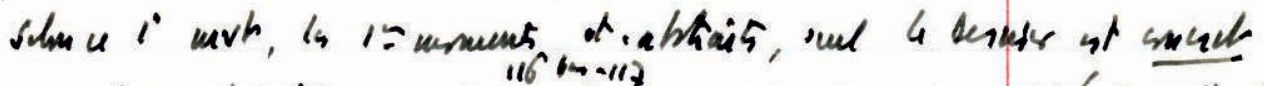

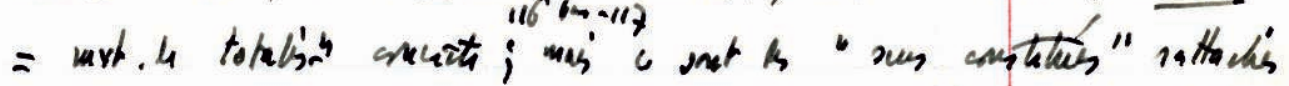

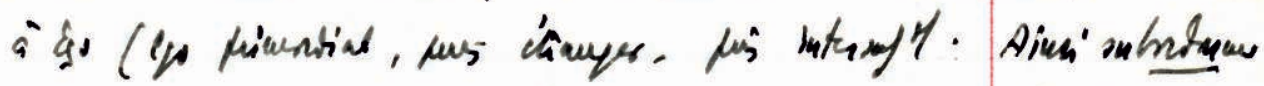

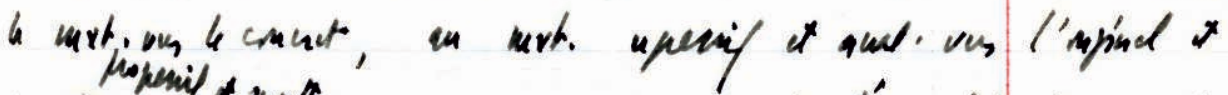

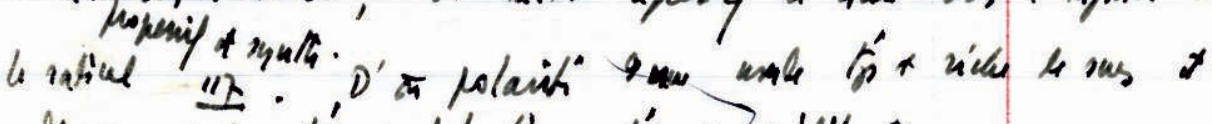

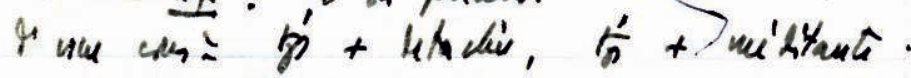

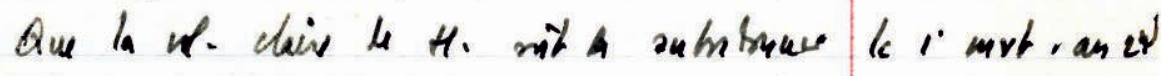


pouvons lire cela dans les notes manuscrites de «Analogie et intersubjectivité chez Husserl» de 1974 (f. 17748) :

Et pourtant le problème ne peut être résolu sans recours à une sorte - d'analogie. Une perception interprétante ou une interprétation perceptive n'atteint jamais qu'un objet plus complexe, qu'une «chose» (Ding), non un autre sujet, c.a.d. comme moi. L'énigme, c'est bien la réduplication de l'ego dans l'expression $<$ alter $>$ alter ego. C'est cette réduplication qui est analogisante.

Ce passage est repris dans le manuscrit «Phénoménologie hégélienne et phénoménologie husserlienne» avant d'être réinséré presque littéralement dans l'essai «Hegel et Husserl sur l'intersubjectivité 23 ». Nous citons ici le manuscrit (f. 17285-17286) :

Un texte de 1914, le n. 13 du I vol de Iso Kern montre bien comment la symetrie entre la perc. interprétante de la ch. et la perc.n interprétante du comprt.t [comportement] est rompue par la co-position - la Mitsetzung - présentifiante qui ordonne un autre sujet à l'app.ce externe. L'énigme cachée dans l'évidence quotidienne, c'est dans la réduplication de l'ego dans l'expression alter ego. C'est cette reduplic.on qui demande que l'analogie soit remise en chantier.

C'est alors à la suite de la publication des Inédits sur l'intersubjectivité que Ricœur se convainc de la possibilité d'interpréter la constitution comme Auslegung et qu'il affirme sans ambiguïtés la validité de la théorie husserlienne de l'intersubjectivité. Alors que la théorie de l'analogie, jusque dans les années soixante, demeurait sous l'emprise du sens général de la phénoménologie husserlienne, c'est maintenant l'étude de la théorie de la constitution de l'autre qui semble faire revenir Ricœur sur le sens même de la constitution, pour l'affranchir définitivement de la hantise de l'idéalisme et du subjectivisme. Et c'est ainsi que, en réhabilitant cette doctrine transcendantale de l'intersubjectivité, il est possible de retrouver chez Husserl une «instance critique» contre le risque d'hypostase de l'esprit objectif hégélien.

Cette étude génétique, dès lors, ne se restreint pas à la simple reconstitution des étapes ayant abouti à la publication de l'essai «Hegel et Husserl sur l'intersubjectivité». Au contraire, elle révèle plusieurs plans du questionnement philosophique de Ricœur, où deviennent visibles ses hésitations, ses approfondissements et ses changements d'opinion : alors que le texte publié finit inévitablement par les omettre ou les aplanir, l'étude génétique les fait remonter à la surface et nous conduit à réévaluer le rapport complexe et non linéaire que Ricœur entretient avec Hegel et Husserl. Ainsi avons-nous pu voir comment Ricœur, en travaillant sur le thème de la volonté, redécouvre et accentue la puissance de la philosophie hégélienne, qui prend la place de la critique kantienne dans la mise en évidence des limites de l'intentionnalité. Nous avons essayé de montrer comment ce remplacement implique, en parallèle, une révision de la philosophie husserlienne, sans laquelle il serait impossible d'inscrire dans l'intentionnalité l'esprit objectif hégélien. Par rapport aux positions exprimées par Ricœur dans les années cinquante, la genèse de cet essai nous oblige dès lors à prendre au sérieux la référence à Hegel, en tant qu'elle structure intimement le cheminement de pensée de Ricœur, et à mesurer toute l'ambivalence du rapport que celui-ci noue avec la phénoménologie de Husserl.

23. Voir P. Ricœur, «Hegel et Husserl sur l'intersubjectivité», art. cité, p. 293.

Chiara Pavan est doctorante à l'Università del Salento (Italie) et à l'Université Paris IV Sorbonne, où elle enseigne aussi la philosophie. Sa thèse porte sur l'ontologie dans la pensée d'Emmanuel Levinas et de Paul Ricœur. Elle a publié des articles sur les implications ontologiques de l'attestation et du symbole chez Ricœur, sur la signification et le langage chez Levinas, ainsi que sur la question de la technique chez Günther Anders.

chiarapav@gmail.com

Roberta PiCARDi est professeure à l'Université del Molise (Italie) et Membre du Conseil Scientifique du Fonds Ricœur. Ses travaux portent sur la philosophie classique allemande et sur son héritage dans la philosophie contemporaine. Elle a publié notamment une monographie sur la philosophie de l'histoire de Fichte (Il concetto e la storia, Bologna, il Mulino, 2009) et dirigé le numéro thématique de la Revue des sciences philosophiques et théologiques «Paul Ricœur : de la volonté à l'action» (t. 99, 2015/4).

roberta_picardi@virgilio.it 


\section{"Hegel et Husserl sur l'intersubjectivité": la genèse longue d'un écrit de circonstance}

Bien qu'il s'agisse d'un écrit de circonstance, l'essai de Ricœur «Hegel et Husserl sur l'intersubjectivité » est l'issue d'une gestation longue, que cet article se propose de reconstruire. Cette gestation longue présente en effet un double intérêt. Tout d'abord, elle nous donne un aperçu significatif du corps à corps spéculatif avec d'autres auteurs, tels que Hegel et Husserl, dont se nourrissent sans cesse l'écriture et la pensée de Ricœur. En outre, ce parcours génétique permet de suivre, pas à pas, l'itinéraire qui conduit Ricœur à abandonner la conception de l'intersubjectivité défendue dans les écrits des années 1950, dans lesquels il se réclamait principalement de Kant, selon une orientation à la fois antihégélienne et anti-husserlienne. C'est le mouvement non linéaire sous-tendant ce tournant que nous visons à mettre à jour, en nous concentrant sur deux segments de la genèse : les traces du questionnement qui aboutit à la conception du premier «schéma» de l'essai, et les notes sur la «Cinquième méditation» de Husserl.

Though this is an occasional text, Ricoeur's essay "Hegel and Husserl on Intersubjectivity" is the result of a long gestation, which this article intends to reconstruct. Indeed, this long gestation presents a twofold interest. First, it gives us a significant glimpse of this speculative battle with other authors such as Hegel and Husserl that have constantly nourished Ricoeur's writing and thought. Moreover, this genetic process enables us to follow, step by step, the path that leads Ricoeur to abandon the conception of intersubjectivity he defended in his 1950 writings, in which he aligned himself mainly with Kant, according to an orientation both anti-Hegelian and anti-Husserlian. We will try to show the nonlinear movement that underlies this shift, by focusing on two segments of the genesis: the marks of the investigation which led to the conception of the essay's first "scheme", and the notes on Husserl's "Fifth Meditation".

Obwohl es sich um eine Gelegenheitsschrift handelt, ist Ricœurs Aufsatz „Hegel und Husserl über Intersubjektivität“ das Ergebnis eines langen Entstehungsprozesses, die der gegenwärtige Artikel zu rekonstruieren versucht. Dieser lange Entstehungsprozess ist in zweierlei Hinsicht interessant. Zum einen bietet er uns ein bedeutendes Beispiel für einen spekulativen Nahkampf mit anderen Autoren, wie Hegel und Husserl, von denen sich Ricœurs Schreiben und Denken beständig nährt. Zum anderen sehen wir im genetischen Parcours Schritt für Schritt, wie Ricœur seine prinzipiell Kantische Konzeption von Intersubjektivität, die er in seinen Schriften der fünfziger Jahre verteidigte, aufgibt, und zwar gemäß einer Orientierung, die zugleich gegen Hegel und gegen Husserl gerichtet ist. Wir versuchen die nicht-lineare Bewegung sichtbar zu machen, welche dieser Wende zugrunde liegt, und konzentrieren uns dabei auf zwei Segmente der Genese: die Spuren jener Fragestellung, die in die Konzeption des ersten „Schemas“ des Aufsatzes mündet, und die Anmerkungen zur „Fünften Meditation" Husserls.

A unque se trata de un artículo de circunstancias, el ensayo de Ricœur "Hegel et Husserl sur l'intersubjetivité" es el resultado de una larga gestación, que este artículo intentará reconstruir. Esta larga gestación presenta, en efecto, un doble interés. Ante todo, nos brinda un ejemplo significativo del combate especulativo con otros autores, como Hegel y Husserl, que nutren permanentemente la escritura y el pensamiento de Ricœur. Por otra parte, ese recorrido genético permite seguir paso a paso el itinerario que lleva a Ricœur a abandonar la concepción de la intersubjetividad sostenida en los escritos de los años cincuenta, en los cuales adhería esencialmente a Kant, siguiendo una orientación a la vez antihegeliana $\mathrm{y}$ antihusserliana. Trataremos de analizar el movimiento no-lineal en el que se asienta ese giro, concentrándonos en dos segmentos de la génesis: las huellas de las interrogaciones que conducen a la concepción del primer "patrón" del ensayo y las notas sobre la "Quinta meditación” de Husserl.

Texto de circunstância, o ensaio de Ricoeur "Hegel e Husserl sobre a intersubjectividade" é o resultado de uma longa gestação, aqui reconstituída e de duplo interesse. Em primeiro lugar, dá-nos um significativo olhar sobre o combate especulativo com outros autores, como Hegel e Husserl, que alimenta a escrita e o pensar de Ricœur. Além disso, este percurso genético faz seguir, passo a passo, a rota que levou Ricœur a abandonar o conceito de intersubjectividade que defendera nos escritos da década de 1950, em que se reclamava de Kant e opunha tanto a Hegel como a Husserl. Um movimento não-linear subjaz a sua reviravolta, o que fica bem evidenciado por dois segmentos da génese: as hesitações que conduzem ao primeiro esboço do ensaio e as notas sobre a "Quinta meditação" de Husserl.

Benché si tratti di uno scritto di circostanza, il saggio di Ricœur "Hegel et Husserl sur l'intersubjectivité" è il risultato di una gestazione lunga, che questo articolo si propone di ricostruire. Questa gestazione lunga presenta infatti un duplice interesse. In primo luogo, ci fornisce un esempio significativo del corpo a corpo speculativo con altri autori, come Hegel e Husserl, che alimentano continuamente la scrittura e il pensiero di Ricœur. Inoltre, questo percorso genetico permette di seguire passo a passo l'itinerario che conduce Ricœur ad abbandonare la concezione dell'intersoggettività difesa negli scritti degli anni Cinquanta, nei quali si rifaceva principalmente a Kant, seguendo un orientamento al tempo stesso anti-hegeliano e anti-husserliano. Quest'articolo vuole mettere in luce il movimento non lineare alla base di questa svolta, soprattutto attraverso l'analisi di due segmenti della genesi : le tracce degli interrogativi che sfociano nella concezione del primo "schema" del saggio, e gli appunti sulla "Quinta meditazione" di Husserl. 